\title{
PALINOESTRATIGRAFIA DA FORMAÇÃO SOLIMÕES: COMPARAÇÃO ENTRE BIOESTRATIGRAFIA TRADICIONAL E O MÉTODO DE ASSOCIAÇÕES UNITÁRIAS
}

\author{
KARINA ELOIZA KACHNIASZ \\ Programa de Pós-Graduação em Geociências, Laboratório de Paleontologia e Palinologia de Mato Grosso, Faculdade \\ de Geociências, UFMT, Av. Fernando Correa da Costa, s/n, Bloco E, Sala 151, 78060-900, Cuiabá, MT, Brasil. \\ karinakachniasz@gmail.com
}

\author{
SILANE APARECIDA FERREIRA DA SILVA-CAMINHA \\ Laboratório de Paleontologia e Palinologia de Mato Grosso, Faculdade de Geociências, UFMT, \\ Av. Fernando Correa da Costa, S/N, Bloco E, Sala 151, 78060-900, Cuiabá, MT, Brasil. \\ silane.silva@gmail.com
}

\begin{abstract}
PALYNOSTRATIGRAPHY SOLIMÕES FORMATION: COMPARISON BETWEEN TRADITIONAL BIOSTRATIGRAPHY AND UNITARY ASSOCIATION METHOD. Palynological analyses were carried out on 48 samples collected in 1AS-31-AM and 1AS-34-AM wells in order to establish a local biostratigraphical framework using qualitative and quantitative methods. Those wells were drilled in the 1970s to investigate the potential of lignite from the Solimões Formation. Our results in traditional palynostratigraphy suggest late Miocene and Pliocene ages. The marker species as Grimsdalea magnaclavata, fenestrate pollen grains, Ladakhipollenites? caribbiensis and Echitricolporites mcneillyi allow to include sections into Psilatricolporites caribbiensis and Echitricolporites-Alnipollenites subzones sensu Lorente. Using Unitary Association method, seven unitary associations were created and four zones indicating similar species composition between wells. Comparisons between the methods indicate that both wells are contemporaneous.
\end{abstract}

Key words: Solimões Formation, biostratigraphy, upper Miocene-Pliocene, Unitary Association.

RESUMO - Análises palinológicas foram realizadas em 48 amostras coletadas nos poços 1AS-31-AM e 1AS-34-AM a fim de se estabelecer um quadro bioestratigráfico local usando métodos bioestratigráficos qualitativos e quantitativos. Os poços foram locados para a pesquisa do potencial linhítico da Formação Solimões, na década de 1970. Nossos resultados em palinoestratigrafia, pelo método tradicional, sugerem idades Neomioceno e Plioceno. As espécies-índice como Grimsdalea magnaclavata, grãos de pólen fenestrados, Ladakhipollenites? caribbiensis e Echitricolporites mcneillyi permitem incluir tais seções dentro das subzonas Psilatricolporites caribbiensis e Echitricolporites-Alnipollenites sensu Lorente. Usando o método de Associações Unitárias, sete associações unitárias e quatro zonas foram criadas indicando uma composição de espécies similares entre os poços. Comparações entre os dois métodos indicam que ambos os poços são contemporâneos.

Palavras-chave: Formação Solimões, bioestratigrafia, Mioceno superior-Plioceno, Associação Unitária.

\section{INTRODUÇÃO}

O surgimento do atual cenário amazônico ocorreu no Neógeno, quando grandes mudanças paleoambientais ocorreram na região, principalmente, em função dos picos de atividade tectônica na margem andina, ao noroeste da plataforma. Nesse período, desenvolveu-se um complexo sistema de ambientes deposicionais continentais, o qual originou a Formação Solimões. Essa unidade litoestratigráfica, por sua vez, possui distribuição estimada em 500 mil km² estendendo-se, em território brasileiro, nas bacias sedimentares do Solimões, do Acre e do Amazonas (Maia et al., 1977).

A principal mudança ao longo do desenvolvimento do sistema amazônico ocorreu no Mesomioceno-Neomioceno, com a inversão da direção de fluxo do sistema fluvial, que passou a drenar para o Oceano Atlântico (Hoorn et al., 1995, 2010; Shephard et al., 2010). Esse evento pode ser sustentado pela origem da foz do Rio Amazonas no Oceano Atlântico no período entre 11.8 e 8.3 Ma (Figueiredo et al., 2009; Gorini et al., 2014).

Dados palinológicos foram usados para estimar a idade de deposição da Formação Solimões por comparação com zoneamentos estabelecidos em países vizinhos, como Colômbia e Venezuela, usando-se métodos tradicionais (Germeraad et al., 1968; Lorente, 1986; Jaramillo et al., 2011). As idades atribuídas para Formação Solimões variam entre Mioceno e início do Plioceno (Hoorn, 1993; Leite, 2006; Leite et al., 2016; Silva-Caminha et al., 2010)

O ambiente de deposição da Formação Solimões é predominantemente lacustre e fluvial, com episódios de 
incursões marinhas registrados por alguns autores no Mioceno inferior e médio/superior (Maia et al., 1977; Hoorn, 1993; Hoorn et al., 1995, 2010; Cozzuol, 2006; Lovejoy et al., 2006; Latrubesse et al., 2007, 2010; Silva-Caminha et al., 2010; Shephard et al., 2010; Linhares et al., 2011; Boonstra et al., 2015).

Informações sobre idade e ambiente ainda são insuficientes ao considerar-se o tamanho da área e a descontinuidade do registro bioestratigráfico. Portanto, este trabalho tem como objetivo estabelecer idade e correlação entre os poços 1AS-34AM e 1AS-31-AM usando dados palinológicos e integrando métodos de bioestratigrafia tradicional e quantitativa.

\section{MATERIAL E MÉTODOS}

Os poços 1AS-34-AM e 1AS-31-AM foram perfurados na porção noroeste do Estado do Amazonas, próximo à fronteira com o Peru (Figura 1). O primeiro está locado à margem do rio Jutaí, coordenadas $05^{\circ} 37^{\prime} \mathrm{S}$ e $69^{\circ} 10^{\prime} \mathrm{O}$ e o segundo, locado à margem do rio Ituí, coordenadas $05^{\circ} 18^{\prime} \mathrm{S}$ e $71^{\circ} 02^{\prime} \mathrm{O}$. A locação desses poços, juntamente com outros 82 poços, foi realizada por duas companhias brasileiras, o Departamento Nacional de Produção Mineral (DNPM) e Companhia de Pesquisa de Recursos Minerais (CPRM), durante a execução do Projeto Carvão no Alto Solimões na década de 70 (Maia et al., 1977).

Para análise palinológica, 54 amostras foram selecionadas, das quais apenas 48 apresentaram resíduo orgânico (23 amostras do poço 1AS-34-AM e o restante do poço 1AS-31AM) (Tabela 1).

A metodologia empregada na preparação química está descrita em Traverse (1988) e consiste no uso de ácido fluorídrico (HF) e ácido clorídrico $(\mathrm{HCl})$ para dissolução de silicatos e carbonatos, respectivamente, e cloreto de zinco para separação por densidade. As lâminas foram montadas com bálsamo de Canadá e lidas em microscópio binocular Nikon E200, com meta de contagem de 300 grãos de pólen e esporos por nível amostrado. No caso de amostras pouco férteis para palinologia, tentou-se chegar a uma contagem mínima de 100 espécimes (Tabela 1).

Para cada espécie foi confeccionado um index card com o desenho do grão, sua descrição morfológica e localização na lâmina em coordenadas de England Finder. Os index cards, assim como as lâminas, estão disponíveis para consulta no Laboratório de Palinologia e Paleontologia da Faculdade de Geociências da Universidade Federal de Mato Grosso.

A consulta à literatura especializada auxiliou na identificação das espécies (Germeraad et al., 1968; Lorente, 1986; Muller et al., 1987; Hoorn, 1993; Leite, 2006; Silva-Caminha et al., 2010; Jaramillo \& Rueda, 2013). Posteriormente, fotomicrografias foram feitas na câmera Leica ICC50 HD acoplada ao microscópio Leica DM750. Os diagramas polínicos da Figura 2 foram processados no software C2 (Juggins, 2007).

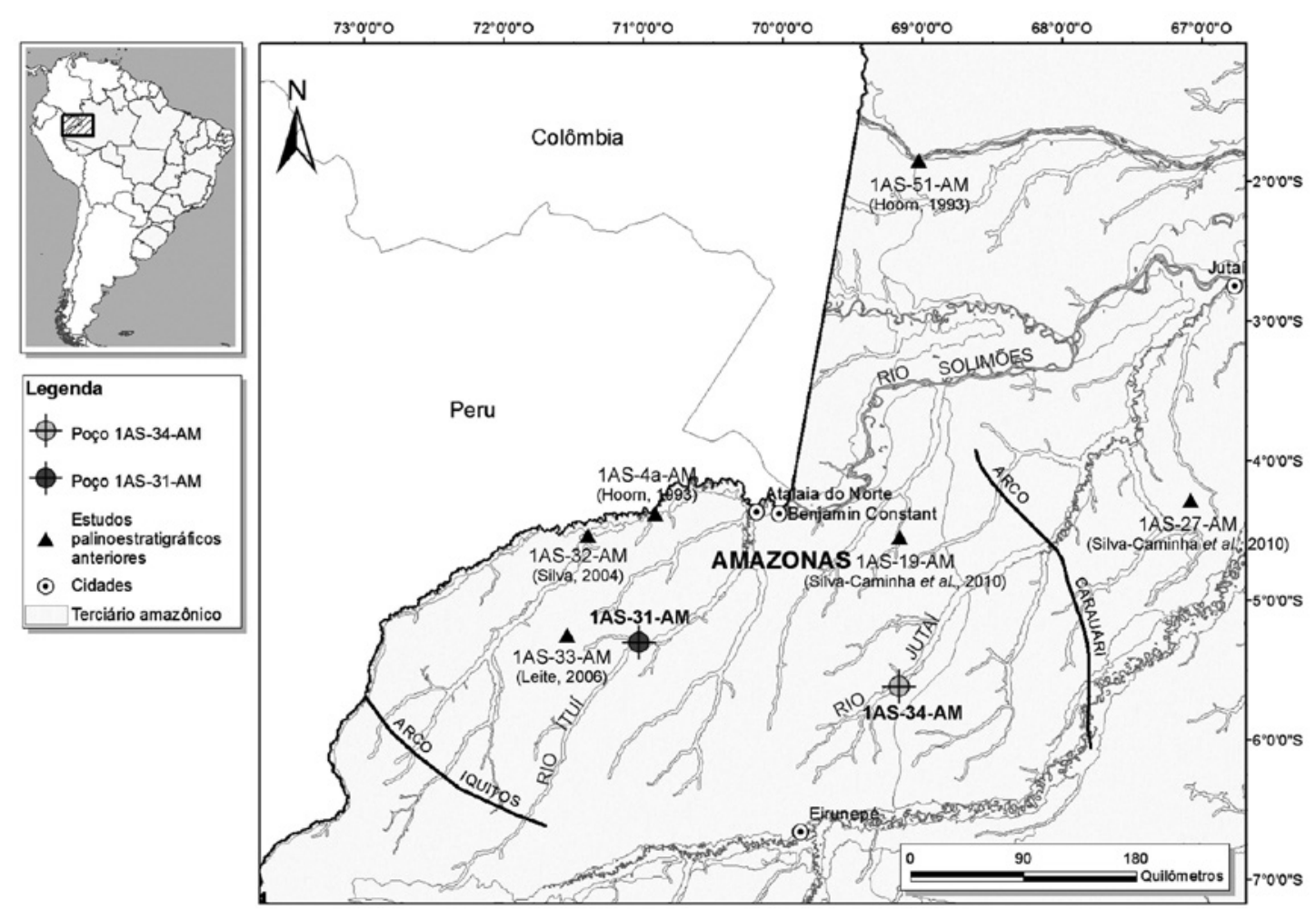

Figura 1. Mapa de localização dos poços 1AS-31-AM e 1AS-34-AM. Triângulos mostram os outros poços já estudados na região por Hoorn (1993), Silva (2004), Leite (2006) e Silva-Caminha et al. (2010).

Figure 1. Location map of the wells 1AS-31-AM and 1AS-34-AM. Triangles indicate wells studied previously by Hoorn (1993), Silva (2004), Leite (2006) and Silva-Caminha et al. (2010). 
Tabela 1. Lista com os níveis amostrados e a quantidade de espécimes contados.

Table 1. List of the samples and specimens counted.

\begin{tabular}{|c|c|c|c|}
\hline & \multicolumn{3}{|c|}{ 1AS-34-AM } \\
\hline & Amostras (m) & Lâminas & Espécimes \\
\hline 1 & 25 & $\bullet \bullet$ & 86 \\
\hline 2 & 29 & $\bullet \bullet$ & 6 \\
\hline 3 & 37 & - & 319 \\
\hline 4 & 50 & $\bullet \bullet$ & 25 \\
\hline 5 & 56 & $\bullet \bullet$ & 30 \\
\hline 6 & 63 & $\bullet \bullet$ & 28 \\
\hline 7 & 67.1 & $\bullet \bullet$ & 15 \\
\hline 8 & 67.4 & $\bullet$ & 315 \\
\hline 9 & 73 & $\bullet \bullet$ & 19 \\
\hline 10 & 79.3 & $\bullet \bullet$ & 13 \\
\hline 11 & 84.7 & $\bullet$ & 347 \\
\hline 12 & 88.28 & $\bullet$ & 316 \\
\hline 13 & 90.95 & $\bullet \bullet \bullet$ & 110 \\
\hline 14 & 103.6 & $\bullet \bullet$ & 307 \\
\hline 15 & 113.9 & $\bullet$ & 306 \\
\hline 16 & 115.25 & $\bullet \bullet$ & 4 \\
\hline 17 & 128.54 & $\bullet$ & 304 \\
\hline 18 & 133.89 & $\bullet$ & 319 \\
\hline 19 & 149.35 & $\bullet \bullet \bullet$ & 46 \\
\hline 20 & 167.7 & $\bullet \bullet$ & 28 \\
\hline 21 & 168.3 & $\bullet$ & 325 \\
\hline 22 & 189.25 & $\bullet \bullet$ & 3 \\
\hline 23 & 199.95 & $\bullet$ & 321 \\
\hline & Total & & 3.592 \\
\hline
\end{tabular}

\begin{tabular}{|c|c|c|c|}
\hline & \multicolumn{3}{|c|}{ 1AS-31-AM } \\
\hline & Amostras (m) & Lâminas & Espécimes \\
\hline 1 & 20.53 & $\bullet \bullet$ & 11 \\
\hline 2 & 20.61 & $\bullet \bullet$ & 38 \\
\hline 3 & 25.86 & - & 306 \\
\hline 4 & 50.5 & $\bullet$ & 315 \\
\hline 5 & 56.5 & $\bullet \bullet$ & 314 \\
\hline 6 & 58 & $\bullet \bullet \bullet \bullet$ & 312 \\
\hline 7 & 65.75 & $\bullet$ & 300 \\
\hline 8 & 89 & $\bullet \bullet$ & 30 \\
\hline 9 & 90.6 & $\bullet$ & 309 \\
\hline 10 & 135 & $\bullet$ & 30 \\
\hline 11 & 138.65 & $\bullet \bullet$ & 22 \\
\hline 12 & 160 & $\bullet \bullet$ & 4 \\
\hline 13 & 170.4 & $\bullet \bullet \bullet$ & 125 \\
\hline 14 & 173.6 & $\bullet$ & 312 \\
\hline 15 & 174.53 & $\bullet \bullet$ & 284 \\
\hline 16 & 175 & $\bullet \bullet$ & 86 \\
\hline 17 & 180.95 & $\bullet \bullet$ & 16 \\
\hline 18 & 183 & - & 8 \\
\hline 19 & 187.15 & $\bullet \bullet \bullet$ & 267 \\
\hline 20 & 197.15 & $\bullet$ & 32 \\
\hline 21 & 213 & $\bullet \bullet$ & 33 \\
\hline 22 & 230 & $\bullet \bullet$ & 62 \\
\hline 23 & 257 & $\bullet$ & 33 \\
\hline 24 & 279 & $\bullet$ & 324 \\
\hline 25 & 287.15 & $\bullet \bullet$ & 21 \\
\hline & Total & & 3.594 \\
\hline
\end{tabular}

Com relação aos estudos de cunho bioestratigráfico, dois métodos foram usados: o tradicional e o quantitativo. Ambos os métodos fazem uso do conteúdo fossilífero nos pacotes sedimentares para o estabelecimento de biozonas. Na bioestratigrafia tradicional, a presença e/ou ausência de espécies geram diversos tipos de biozonas, que refletem eventos específicos no tempo, como abundância, o surgimento ou a extinção de determinada espécie. Para esta, os trabalhos de Germeraad et al. (1968), Lorente (1986), Muller et al. (1987) e Jaramillo et al. (2011) foram consultados. O segundo método foi adotado a fim de diminuir o efeito da descontinuidade dos dados bioestratigráficos em camadas sedimentares, causados por fatores diversos, mas principalmente devido ao controle ecológico das espécies no ambiente. Aqui se utilizou o método de Associações Unitárias (UA - Unitary Association), que consiste na aplicação de um modelo determinístico aos dados, visando obter Zonas de Distribuição Concorrente (Concurrent-range Zones), onde cada zona seja distinta e tenha o número máximo de espécies coexistentes (Savary \& Guex, 1999).
Toda zona estabelece-se a partir de UA's reprodutíveis, as quais possuem espécies e/ou pares de espécies características que permitem identificá-las em diferentes localidades. Estes são formados por espécies que tiveram seu primeiro aparecimento e último aparecimento na UA.

Relações de superposição e coexistência de 48 espécies foram utilizadas na construção das UA's. A eliminação do restante seguiu critérios bioestratigráficos, os quais apontam como marcadores os taxa que apresentam ampla distribuição geográfica e curta distribuição no tempo, principalmente. Portanto, foram excluídas as espécies endêmicas (registradas em apenas um poço) e com ampla distribuição vertical em ambos os poços, além dos grupos identificados somente em nível de gênero e informais "sp. X, W, 1, 2, etc.". Também foram excluídos os níveis com contagem inferior a 250 espécimes. Os dados foram processados no software PAST versão 2.17 (Hammer et al., 2001).

As descrições litológicas das seções foram retiradas dos dados originais do Projeto Carvão no Alto Solimões (Maia et al., 1977) e estão ilustradas na Figura 2. 


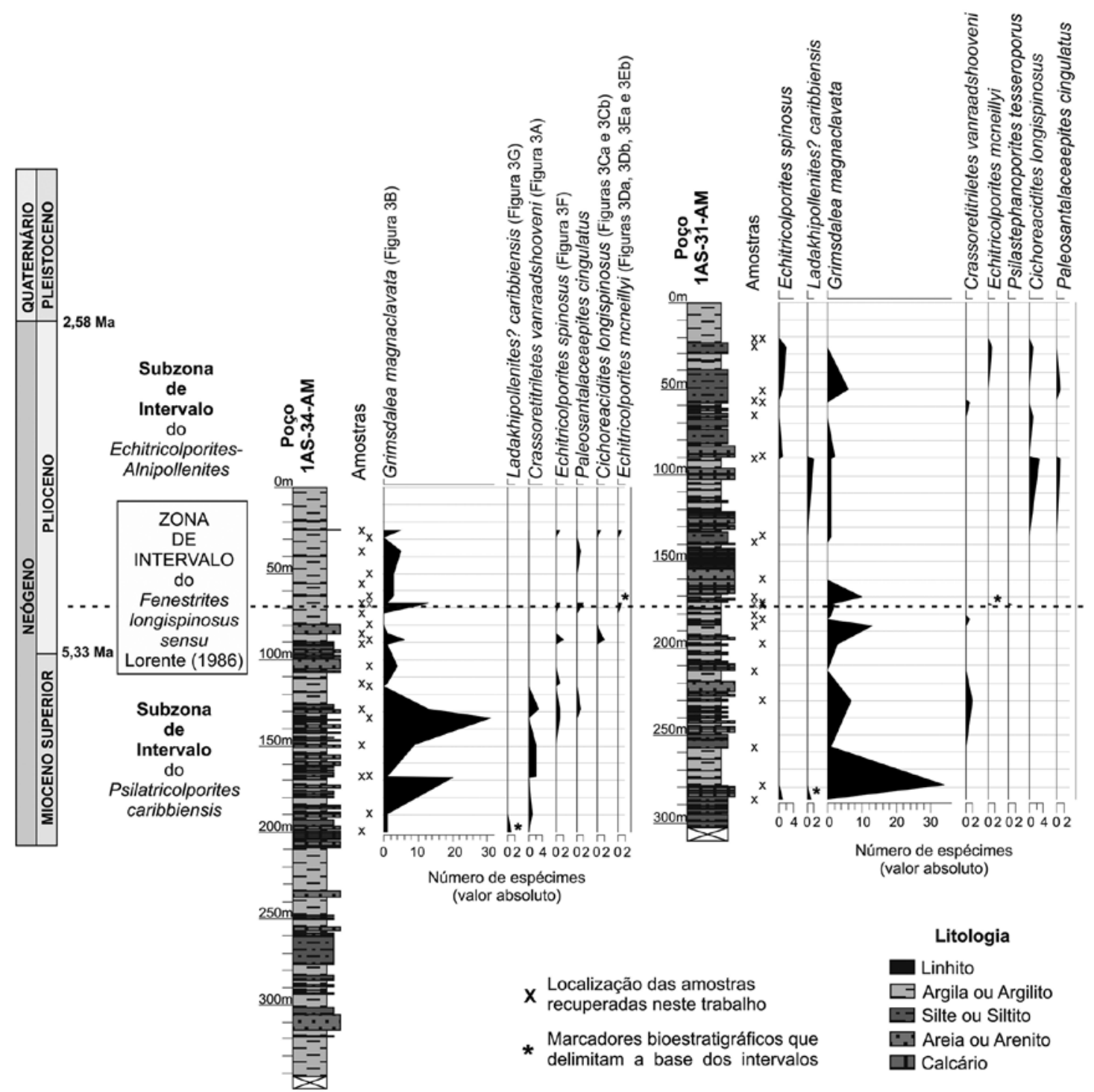

Figura 2. Biozoneamento nos testemunhos 1AS-31-AM e 1AS-34-AM segundo arcabouço bioestratigráfico de Lorente (1986) e correlação entre eles.

Figure 2. Biozonation of the 1AS-31-AM e 1AS-34-AM using Lorente (1986) framework and their correlations.

\section{RESULTADOS}

\section{Bioestratigrafia tradicional}

A presença de Ladakhipollenites? caribbiensis (nas amostras mais basais em ambos os poços permite afirmar idade mínima de Neomioceno, podendo estender-se até o Plioceno (Tabela 2). Outros marcadores bioestratigráficos do Mioceno também ocorrem associados a esta espécie, como Grimsdalea magnaclavata, Crassoretitriletes vanraadshooveni e Echitricolporites spinosus (Figura 2). Nos poços 1AS-34-AM e 1AS-31-AM este intervalo vai de 199,95 a $67,4 \mathrm{~m}$ e de 287,15 a $174,53 \mathrm{~m}$, respectivamente.

Echitricolporites mcneillyi é a única espécie aqui registrada que apresenta o "first appearance datum" (FAD) posterior ao de Ladakhipollenites? caribbiensis, indicando idade pliocênica, ou muito próxima a este limite, às seções estudadas (Germeraad et al., 1968; Lorente, 1986; Muller et al., 1987). Portanto, seus registros nas profundidades de $67,40 \mathrm{~m}$ do poço 34 e 174,53 $\mathrm{m}$ do poço 31 apontam aquela idade aos pacotes acima destes níveis (Figura 2; Tabela 2).

Germeraad et al. (1968) atribuíram o limite MiocenoPlioceno ao FAD de Echitricolporites mcneillyi devido a ausência de outros microfósseis indicativos do Plioceno. Posteriormente, Lorente (1986) e Muller et al. (1987) sugeriram Plioceno para o FAD da espécie. Certa cautela, porém, aqui é tomada em relação à idade pleistocênica apresentada em Jaramillo et al. (2011), com 1,56 Ma, pois difere muito dos outros resultados apontados pela literatura. Do mesmo modo, Echitricolporites mcneillyi ocorre aqui associada a Grimsdalea magnaclavata, extinta durante o Pleistoceno e nenhum marcador desta idade, como Alnipollenites verus, foi observado (Tabela 2). 
Tabela 2. Lista dos principais marcadores bioestratigráficos da Amazônia setentrional e suas respectivas idades de surgimento e extinção. Abreviações: FAD, first appearance datum; LAD, last appearance datum.

Table 2. List of the main biostratigraphic markers from northern Amazon and the ages of the events. Abbreviations: FAD, first appearance datum; LAD, LAD, last appearance datum.

\begin{tabular}{|c|c|c|c|}
\hline Marcadores & FAD & LAD & Autores \\
\hline \multirow{3}{*}{ Alnipollenites verus Potonie, 1931} & Plioceno/Pleistoceno & - & Germeraad et al. (1968) \\
\hline & Pleistoceno & - & Lorente (1986) \\
\hline & Quaternário & - & Muller et al. (1987) \\
\hline \multirow{4}{*}{$\begin{array}{l}\quad \text { Echitricolporites mcneillyi } \\
\text { Germeraad, Hopping \& Muller, } 1968\end{array}$} & Mioceno/Plioceno & - & Germeraad et al. (1968) \\
\hline & Plioceno & - & Lorente (1986) \\
\hline & Plioceno & - & Muller et al. (1987) \\
\hline & Pleistoceno (1.56 Ma) & Pleistoceno (0.78 Ma) & Jaramillo et al. (2011) \\
\hline \multirow{3}{*}{$\begin{array}{l}\text { Ladakhipollenites? caribbiensis } \\
\text { (Muller } \text { et al., 1987) }\end{array}$} & $\begin{array}{l}\text { Mioceno superior } \\
\text { ao Plioceno inferior }\end{array}$ & - & Lorente (1986) \\
\hline & Plioceno & - & Muller et al. (1987) \\
\hline & Mioceno superior (10.36 Ma) & Mioceno superior (10.36 Ma) & Jaramillo et al. (2011) \\
\hline \multirow{3}{*}{$\begin{array}{c}\text { Fenestrites spinosus } \\
\text { Van der Hammen, } 1956 \mathrm{~b}\end{array}$} & Mioceno a Plioceno & - & Regali et al. (1974) \\
\hline & Mioceno superior & - & Lorente (1986) \\
\hline & Mioceno médio (12.7 Ma) & Pleistoceno (0.24 Ma) & Jaramillo et al. (2011) \\
\hline \multirow{2}{*}{$\begin{array}{c}\text { Cichoreacidites longispinosus } \\
\text { (Lorente, 1986) }\end{array}$} & Mioceno superior & - & Lorente (1986) \\
\hline & Mioceno superior (10.06 Ma) & Pleistoceno (1.18 Ma) & Jaramillo et al. (2011) \\
\hline \multirow{4}{*}{$\begin{array}{l}\text { Echitricolporites spinosus } \\
\text { Van der Hammen, } 1956 \mathrm{~b}\end{array}$} & Mioceno & Plioceno & Regali et al. (1974) \\
\hline & $\sim$ Mioceno superior & - & Muller et al. (1987) \\
\hline & Mioceno superior & - & Lorente (1986) \\
\hline & Mioceno inferior (17.41) & Pleistoceno (0.14 Ma) & Jaramillo et al. (2011) \\
\hline \multirow{4}{*}{$\begin{array}{l}\text { Grimsdalea magnaclavata } \\
\text { Germeraad, Hopping \& Muller, } 1968\end{array}$} & Mioceno inferior ao médio & Pleistoceno & Germeraad et al. (1968) \\
\hline & Mioceno & - & Regali et al. (1974) \\
\hline & Mioceno médio ao superior & Pleistoceno & Lorente (1986) \\
\hline & Mioceno inferior (16.09 Ma) & Plioceno (3.4 Ma) & Jaramillo et al. (2011) \\
\hline \multirow{5}{*}{$\begin{array}{l}\text { Crassoretitriletes vanraadshooveni } \\
\text { Germeraad, Hopping \& Muller, } 1968\end{array}$} & Mioceno inferior & - & Germeraad et al. (1968) \\
\hline & Mioceno inferior & - & Regali et al. (1974) \\
\hline & Mioceno médio & - & Lorente (1986) \\
\hline & حMioceno médio & - & Muller et al. (1987) \\
\hline & Mioceno médio (14.18 Ma) & Plioceno (3.4 Ma) & Jaramillo et al. (2011) \\
\hline
\end{tabular}

Destaca-se também que estes são os primeiros achados de Echitricolporites mcneillyi (Figura 3), marcador bioestratigráfico do Plioceno, na Formação Solimões, sendo apenas registrados nas formações Las Piedras e Quiriquire, Catshill, da Venezuela e Trinidad (Germeraad et al., 1968; Lorente, 1986; Muller et al., 1987).

Desta forma, ambos os poços podem ser divididos em dois intervalos: um mais basal, constituído pela presença de Ladakhipollenites? caribbiensis; e outro, superior, constituído por todo o pacote sedimentar acima do primeiro registro do Echitricolporites mcneillyi. Ao aplicar o modelo de biozoneamento pode-se afirmar que os intervalos podem ser incluídos na Zona de Intervalo do Fenestrites longispinosus sensu Lorente (1986) (Figura 2).

O intervalo basal do poço 34 (de 199,95 a 67,4 m) corresponde a Subzona de Intervalo Psilatricolporites caribbiensis sensu Lorente (1986). O intervalo superior (67,4 a $25 \mathrm{~m}$ ) corresponde a Subzona de Intervalo Echitricolporites-
Alnipollenites sensu Lorente (1986), a qual possui o limite inferior determinado pelo primeiro aparecimento de Echitricolporites mcneillyi.

De forma semelhante, o intervalo basal do poço 31 (287,15 a 174,53 m) corresponde a Subzona de Intervalo Psilatricolporites caribbiensis sensu Lorente (1986), enquanto o intervalo superior (174,53 a 20,53 m) corresponde a Subzona de Intervalo Echitricolporites-Alnipollenites sensu Lorente (1986).

A diferença de mais de $100 \mathrm{~m}$ desta última subzona em ambos os poços pode ser atribuída a dois fatores principais: a distância geográfica entre os poços, que corresponde a aproximadamente $215 \mathrm{~km}$; ou a ausência de análises palinológicas nos $100 \mathrm{~m}$ mais basais do poço 1AS-34-AM, onde a Subzona de Intervalo Echitricolporites-Alnipollenites sensu Lorente (1986) inicia-se mais próxima ao topo que no poço 1AS-31-AM. Amostras deste intervalo não foram recuperadas. 


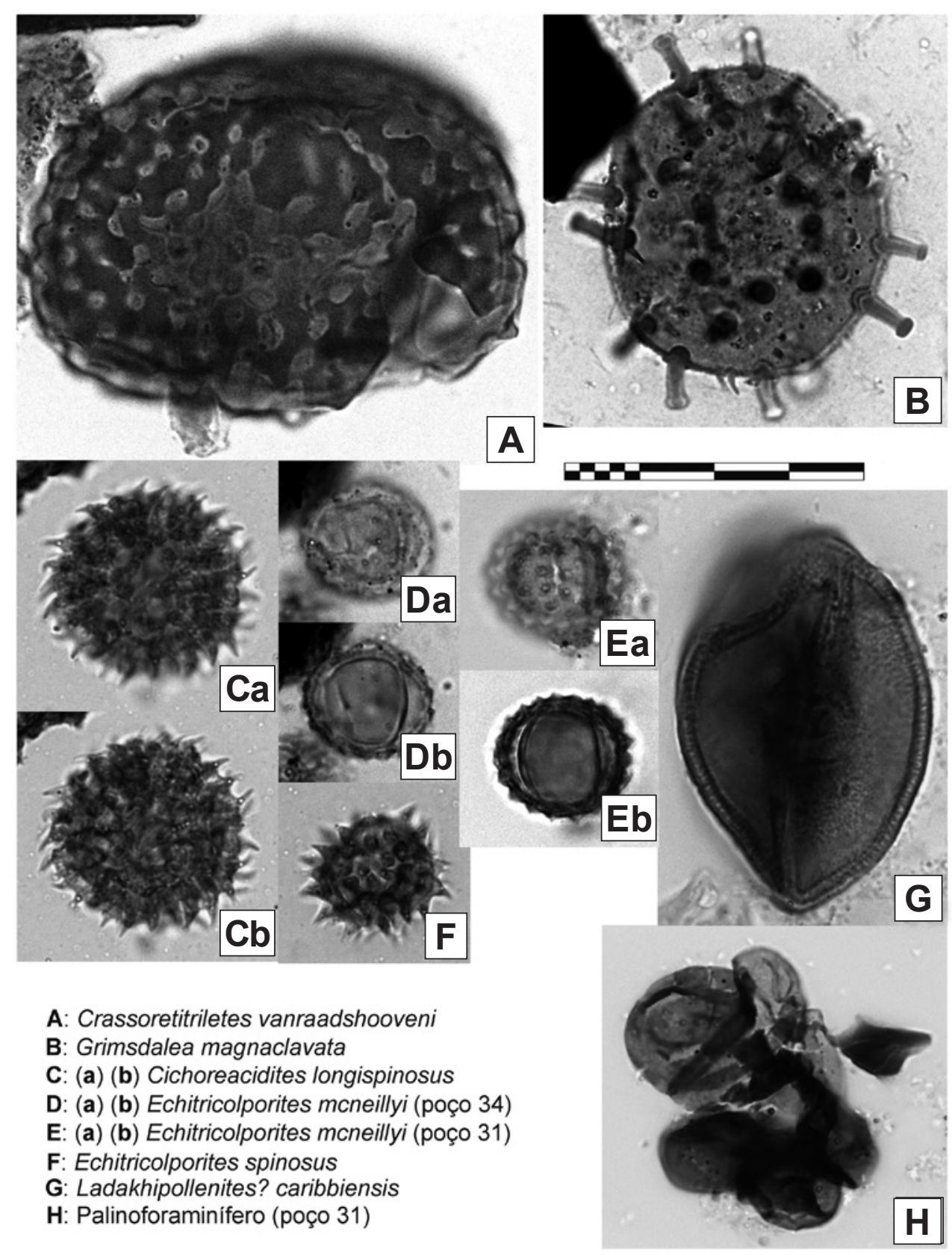

Figura 3. Principais marcadores bioestratigráficos para o Mioceno e Plioceno da Amazônia e um palinoforaminífero. Escala $=40$ um.

Figure 3. Main key species of the Miocene and Pliocene of the Amazonia and a forams record. Scale bar $=40$ um.

\section{Bioestratigrafia quantitativa}

Sete Associações Unitárias (Figuras 4A,D), sete cliques máximos (das profundidades 56,5; 58; e 173,6 m do poço 31 ; e $37 ; 67,4 ; 168,3$; e $199,95 \mathrm{~m}$ do poço 34 ) e 14 horizontes residuais foram obtidos nessa análise. As UA's geradas apresentaram 21 espécies e/ou pares de espécies características, as quais estão listadas esquematicamente na Tabela 3.

Segundo Guex (1991) uma biozona pode ser estabelecida quando se verifica a reprodutibilidade de determinada associação unitária nas seções analisadas, ou seja, quando ocorre uma ampla distribuição geográfica da UA. Logo, o estabelecimento de um biozoneamento em determinadas áreas necessita da presença de UA's com ampla continuidade lateral. A descontinuidade do registro fossilífero, entretanto, dificulta a aplicabilidade desta teoria, sendo então sugerida pelo mesmo autor a união, ou soma, das associações unitárias pouco reprodutíveis segundo critérios específicos, como o índice de similaridade (D) entre as UA's (Figura 4C). Desta forma, amplia-se o número de espécies características de cada UA, ou Zonas no caso, e consequentemente as possibilidades de registro das mesmas.

As UA's 2, 4, 5 e 6 apresentaram baixa reprodutibilidade. Espécies ou pares de espécies característicos da UA-4 não 
Tabela 3. Espécies e/ou pares de espécies característicos de cada Associação Unitária (UA) e/ou Zona. *Silva-Caminha et al. (2010).

Table 3. Species or pair of species of each Unitary Association (UA) or zone. *Silva-Caminha et al. (2010).

\begin{tabular}{|c|c|c|}
\hline ZONAS & UA's & Elementos característicos \\
\hline \multirow{4}{*}{ D } & 7 & Pteridaceoisporis 'lobatus' \\
\hline & & Bombacacidites araracuarensis - Malvacipolloides? sp. 3 * \\
\hline & 6 & Bombacacidites araracuarensis - Tetracolporopollenites transversalis \\
\hline & & Bombacacidites araracuarensis - Bombacacidites fossulatus \\
\hline \multirow{8}{*}{$\mathrm{C}$} & 5 & Rhoipites hispidus - Paleosantalaceaepites $\mathrm{sp} .1 *$ \\
\hline & 5 & Echinatisporis muelleri-Paleosantalaceaepites sp. $1 *$ \\
\hline & & Bombacacidites fossulatus - Multimarginites vanderhammeni \\
\hline & & Polypodiisporites scabraproximatus - Multimarginites vanderhammeni \\
\hline & 4 & Malvacipolloides sp. $2 *$ - Multimarginites vanderhammeni \\
\hline & 4 & Bombacacidites fossulatus - Margocolporites vanwijhei \\
\hline & & Polypodiisporites scabraproximatus - Margocolporites vanwijhei \\
\hline & & Malvacipolloides sp.2* - Margocolporites vanwijhei \\
\hline \multirow{8}{*}{ B } & 3 & Crassoretitriletes vanraadshooveni \\
\hline & & Corsinipollenites oculusnoctis - Siltaria dilcheri \\
\hline & & Bombacacidites baculatus - Siltaria dilcheri \\
\hline & & Retitriletes altimuratus - Siltaria dilcheri \\
\hline & 2 & Echitricolporites spinosus - Siltaria dilcheri \\
\hline & & Crassiectoapertites columbianus - Siltaria dilcheri \\
\hline & & Ranunculacidites operculatus - Siltaria dilcheri \\
\hline & & Polypodiisporites? planus - Siltaria dilcheri \\
\hline A & 1 & Ladakhipollenites? caribbiensis \\
\hline
\end{tabular}

foram identificados no poço 34 , sendo registrados apenas no poço 31. Enquanto as UA's 2, 5 e 6 não foram verificadas no poço 31 (Figura 4B). Assim, união de UA's foi feita segundo o índice de semelhança entre o grupo de espécies constituintes de cada UA, e de modo que estas pudessem ser registradas em ambos os poços. Portanto, quatro zonas puderam ser estabelecidas. São elas: Zona A (UA-1), Zona B (UA's 2 e 3), Zona C (UA's 4 e 5) e Zona D (UA's 6 e 7) (Figura 4E).

\section{Comparações entre os métodos bioestratigráficos e discussões}

Estudos palinoestratigráficos que utilizam o método tradicional indicam idades que variam do Eomioceno ao Neomioceno/Plioceno para outros cinco poços da Formação Solimões (1AS-4a-AM, 1AS-32-AM, 1AS-33-AM 1AS-19AM e 1AS-27-AM) (Hoorn, 1993; Leite, 2006; Silva, 2004; Silva-Caminha et al., 2010). Aqui se registra a presença de Echitricolporites mcneillyi pela primeira vez nesta unidade, indicando Plioceno para o topo da Formação Solimões. Isso não contraria necessariamente as hipóteses de ausência de deposição para este período na Amazônia, mas apoia-se na justificativa de Gorini et al. (2014), em que depósitos de diferentes períodos podem ter se preservado no leito de vales incisos, durante os períodos de alta taxa de erosão e transporte da bacia para a foz. Portanto, tal fato pode explicar o registro de idades mais jovens para aquele ambiente.

Linhares et al. (2011) identificaram, por meio de análises micropaleontológicas no poço $1 \mathrm{AS}-31-\mathrm{AM}$, uma mistura de táxons marinhos, transicionais e não-marinhos no intervalo de 174,9 a 170,9 m, sugerida como uma fase continental com influência marinha. Dentre os microrganismos registrados estão foraminíferos bentônicos e planctônicos, além de briozoários. Nas análises palinológicas aqui descritas foi identificado apenas um espécime de palinoforaminífero neste mesmo intervalo, na amostra de 174,53 m (Figura 2H), corroborando, portanto, a hipótese de Linhares et al. (2011) de um evento de incursão marinha, ao menos episódica, no interior da Amazônia durante o Neógeno. Nenhum registro de dinoflagelado foi observado. O registro de apenas um palinoforaminífero pode ser explicado pelo método de preparação das amostras que usou em várias etapas a centrifugação, podendo ter prejudicado a preservação de dinoflagelados. Apoiando em estudos palinológicos do poço 1AS-4a-AM, Linhares et al. (2011) dataram a incursão como mesomiocênica. Aqui, o limite entre as biozonas ocorre justamente no mesmo nível em que Linhares et al. (2011) sugerem a fase transicional, sendo, portanto, o episódio de incursão mais recente datada aqui de idade pliocênica.

Outros registros de incursões marinhas já foram indicados por Hoorn (1993) durante o Eomioceno e Meso/Neomioceno. Recentemente, Hoorn et al. (2010), Shephard et al. (2010) e Gorini et al. (2014) corroboraram com as incursões marinhas na porção central amazônica entre o Mioceno inferior e médio.

As espécies que delimitaram as biozonas nos poços 34 e 31 também foram analisadas sob o método quantitativo. Ladakhipollenites? caribbiensis que delimita a base da Zona de Intervalo de Fenestrites longispinosus é registrado por meio deste método apenas na Zona A (UA-1) e considerado, 
(D)

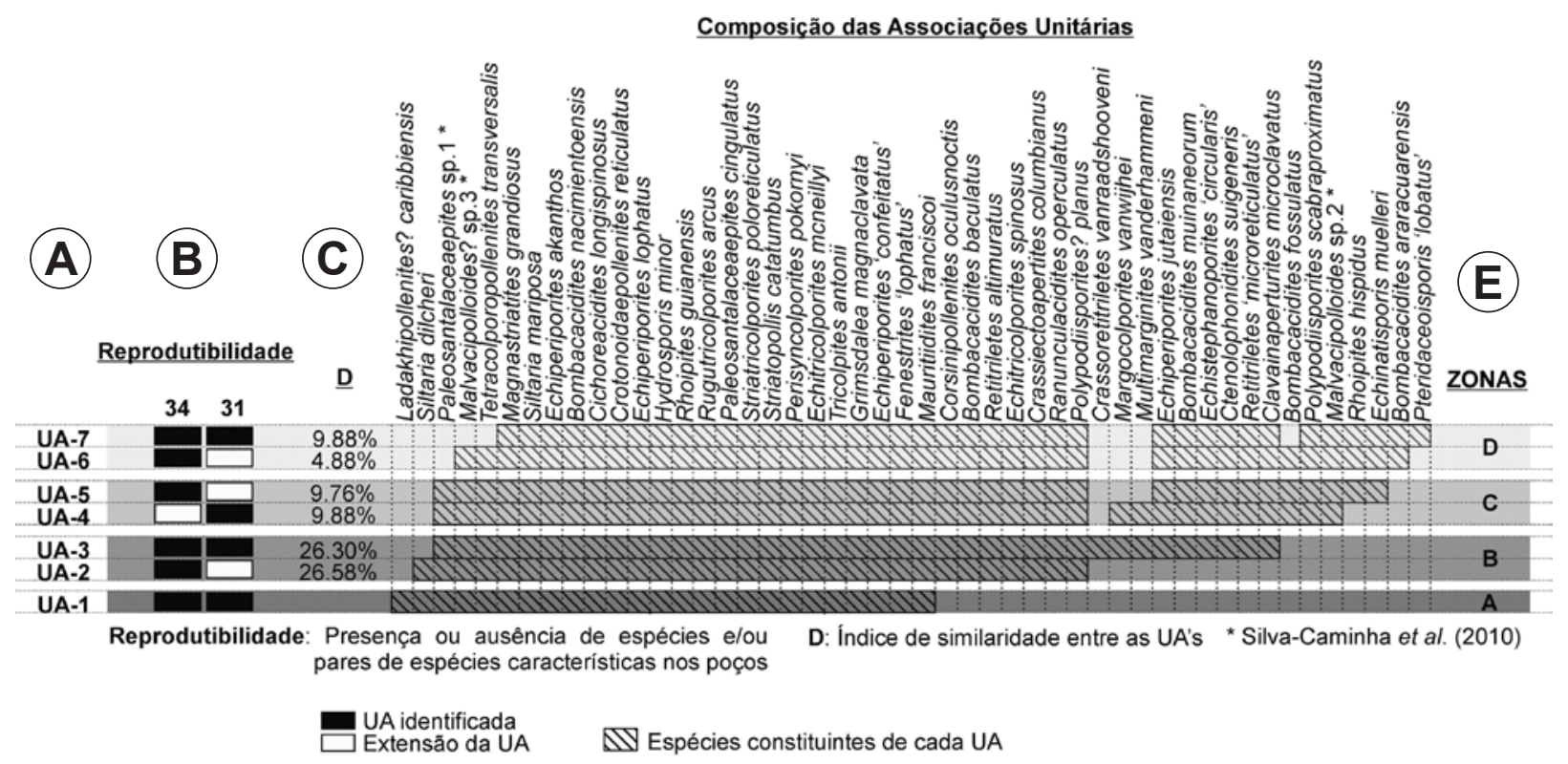

Figura 4. Relação das Associações Unitárias (1 a 7, da mais antiga para a mais recente) estabelecidas pela análise dos dados bioestratigráficos dos poços 34 e 31 , e suas respectivas espécies (linha) assinaladas em quadrados hachurados quando presentes na UA.

Figure 4. List of Unitary association (1 to 7, from older to younger) and their species (row) indicated as hachure square.

portanto, elemento característico dessa zona. Marcadores de zonas de intervalo mais antigas, como Cichoreacidites longispinosus e Grimsdalea magnaclavata, também fazem parte da Zona A, confirmando que tais espécies coexistiram com essa espécie (Figura 4D). Echitricolporites mcneillyi também coexiste com espécies mais antigas, entrando na composição palinológica de todas as zonas estabelecidas; essa espécie, na Associação Unitária ocorre em zonas distintas,

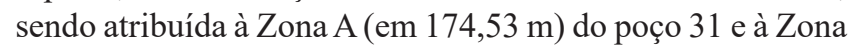
$\mathrm{D}$ (em 67,4 m) do poço 34 .

Apesar dos marcadores utilizados para datar os intervalos nos poços, pela bioestratigrafia tradicional, não serem tão expressivos nas associações unitárias, os dados obtidos por esse método nos poços 34 e 31 mostram que, são contemporâneos entre si e que as espécies predominantes possuem grande amplitude no tempo. A contemporaneidade dos poços também é indicada pela bioestratigrafia tradicional, o que indica a correlação entre os dois poços independente do método utilizado.

Esta é a segunda vez que o método das Associações Unitárias é aplicado nos dados bioestratigráficos da Formação Solimões, sendo utilizado anteriormente por Silva-Caminha et al. (2010) nos poços 1AS-27-AM e 1AS-19-AM. As zonas usadas por aqueles autores não foram aplicadas neste trabalho devido à diferença na composição de espécies características das UA's nos poços 1AS-34-AM e 1AS-31-AM. O ideal, neste caso, seria a união dos dados bioestratigráficos destes quatro poços, para então ser aplicada a técnica das associações unitárias e assim, obter um biozoneamento mais completo. Desta forma, o método das Associações Unitárias poderá ser utilizado futuramente na ampliação do arcabouço bioestratigráfico amazônico. Neste trabalho, espécies reportadas recentemente na literatura, como Siltaria dilcheri, Retitriletes altimuratus e Bombacacidites fossulatus, passam a ganhar uso na bioestratigrafia, constituindo elementos característicos de UA's e Zonas geradas para os poços 34 e 31 (Tabela 3). Para isso, sugere-se aqui a continuidade do uso de tais métodos quantitativos associados à bioestratigrafia tradicional na Formação Solimões.

\section{CONCLUSÕES}

O método tradicional, apoiado no arcabouço bioestratigráfico de Lorente (1986), identificou as biozonas Subzona de Intervalo de Psilatricolporites caribbiensis e Subzona de Intervalo de Echitricolporites-Alnipollenites. A idade atribuída é de Neomioceno e Plioceno.

No poço 34 foram delimitados os intervalos de 199,95 a $67,4 \mathrm{~m}$ e de 67,4 a $25 \mathrm{~m}$, os quais foram atribuídos, respectivamente, as subzonas de intervalo Psilatricolporites caribbiensis e Echitricolporites-Alnipollenites, do Mioceno superior/Plioceno e Plioceno. No poço 31 os intervalos delimitados foram de 287,15 a $174,53 \mathrm{~m}$, atribuído a Subzona de Intervalo de Psilatricolporites caribbiensis, e de 174,53 a 20,53 m, atribuído a Subzona de Intervalo de Echitricolporites-Alnipollenites.

Os resultados obtidos pelo método das Associações Unitárias corroboram aqueles obtidos pela bioestratigrafia tradicional, ao demonstrar a contemporaneidade dos poços da base ao topo. As associações unitárias mostram também que as espécies predominantes nos poços 34 e 31 apresentam grande amplitude vertical, com poucas extinções ao longo do tempo. Este estudo atribui idade pliocênica ao intervalo que apresenta mistura de táxons marinhos e transicionais, 
corroborando com a sugestão em trabalho anterior, indicando idade mais recente do que a literatura propõe para episódios de incursões marinhas na Amazônia.

\section{AGRADECIMENTOS}

O presente trabalho foi realizado com apoio do CNPq, Conselho Nacional de Desenvolvimento Científico e Tecnológico (processo 476020/2013-1). Ao Programa de PósGraduação em Geociências da Faculdade de Geociências da Universidade Federal de Mato Grosso. À F.P.R. Leite e C. Ng pela ajuda na identificação dos palinomorfos. Aos revisores desse artigo, M.A. Carvalho, R. Dino e M.J. Garcia, pelas valiosas sugestões.

\section{REFERÊNCIAS}

Boonstra, M.; Ramos, M.I.F.; Lammertsma, E.I.; Antoine, P.O. \& Hoorn, C. 2015. Marine connections of Amazonia: Evidence from foraminifera and dinoflagellate cysts (early to middle Miocene, Colombia/Peru). Palaeogeography, Palaeoclimatology, Palaeoecology, 417:176-194. doi:10.1016/j. palaeo.2014.10.032

Cozzuol, M.A. 2006. The Acre vertebrate fauna: Age, diversity, and geography. Journal of South American Earth Sciences, 21:185-203. doi:10.1016/j.jsames.2006.03.005

Figueiredo, J.; Hoorn, C. van der Ven, P. \& Soares, E. 2009. Late Miocene onset of the Amazon River and the Amazon deep-sea fan: Evidence from the Foz do Amazonas Basin. Geology, 37:619-622. doi:10.1130/G25567A.1

Germeraad, J.H.; Hopping, C.A. \& Muller, J. 1968. Palynology of Tertiary sediments from tropical areas. Review of Palaeobotany and Palynology, 6:189-348. doi:10.1016/0034-6667(68)90051-1

Gorini, C; Haq, B.U.; dos Reis, A.T.; Silva, C.G.; Cruz, A.; Soares, E. \& Grangeon, D. 2014. Late Neogene sequence stratigraphic evolution of the Foz do Amazonas Basin, Brazil. Terra Nova, 26:179-185. doi:10.1111/ter.12083

Guex, J. 1991. Biochronological correlations. $1^{\text {a }}$ ed. Verlag, Springer, $252 \mathrm{p}$.

Hammer, O.; Harper, D.A.T. \& Ryan, P.D. 2001. Past: Paleontological Statistics Software Package for Education and Data Analysis. Palaeontologia Electronica, 4:1-9. PE 4.1.4A

Hoorn, C. 1993. Miocene incursions and the influence of Andean tectonics on the Miocene depositional history of northwestern Amazonia: results of a palynostratigraphic study. Palaeogeography, Palaeoclimatology, Palaeoecology, 105:267309. doi:10.1016/0031-0182(93)90087-Y

Hoorn, C.; Guerrero, J.; Sarmiento, G.A. \& Lorente, M.A. 1995. Andean tectonics as a cause for changing drainage patterns in Miocene northern South America. Geology, 23:237-240. doi:10.1130/0091-7613(1995)023<0237:ATAACF>2.3.CO

Hoorn, C.; Wesselingh, F.P.; Ter Steege, H.; Bermudez, M.A.; Mora, A.; Sevink, J.; Sanmartín, I.; Sanchez-Meseguer, A.; Anderson, C.L.; Figueiredo, J.P.; Jaramillo, C.; Riff, D.; Negri, F.R.; Hooghiemstra, H.; Lundberg, J.; Stadler, T.; Särkinen, T. \& Antonelli, A. 2010. Amazonia through time: Andean uplift, climate change, landscape evolution, and biodiversity. Science, 330:927-931. doi:10.1126/science.1194585

Jaramillo, C. \& Rueda, M. 2013. A Morphological Electronic Database of Cretaceous-Tertiary and Extant pollen and spores from Northern South America. Disponível em: http://biogeodb. stri.si.edu/jaramillo/palynomorph/; acesso em 09/08/2015.
Jaramillo, C.; Rueda, M. \& Torres, V. 2011. A palynological zonation for the Cenozoic of the Llanos and Llanos Foothills of Colombia. Palynology, 35:46-84. doi:10.1080/01916122.2010.515069

Juggins, S. 2007. C2 Version 1.5: Software for ecological and palaeoecological data analysis and visualization. Newcastle upon Tyne: University of Newcastle.

Latrubesse, E.M.; Cozzuol, M.; Silva-Caminha, S.A.F.; Rigsby, C.A.; Absy, M.L. \& Jaramillo, C. 2010. The Late Miocene paleogeography of the Amazon Basin and the evolution of the Amazon River system. Earth-Science Reviews, 99:99-124. doi:10.1016/j.earscirev.2010.02.005

Latrubesse, E.M.; Silva, S.A.F.; Cozzuol, M. \& Absy, M.L. 2007. Late Miocene continental sedimentation in the southwestern Amazonia and its regional significance: Biotic and geological evidence. Journal of South American Earth Sciences, 23:61-80. doi:10.1016/j.jsames.2006.09.021

Leite, F.P.R. 2006. Palinologia da Formação Solimões, Neógeno da Bacia do Solimões, Estado do Amazonas: Implicações paleoambientais e bioestratigráficas. Instituto de Geociências, Universidade de Brasília, Tese de Doutorado, 128 p.

Leite, F.P.R.; Paz, J.; Carmo, D.A. \& Silva-Caminha, S.A.F. 2016. The effects of the inception of Amazonian transcontinental drainage during the Neogene on the landscape and vegetation of the Solimões Basin, Brazil. Palynology. doi:10.1080/01916 122.2016.1236043

Linhares, A.P.; Ramos, M.I.F.; Gross, M. \& Piller, W.E. 2011. Evidence for marine influx during the Miocene in southwestern Amazonia, Brazil. Geología Colombiana, 36:91-104. doi:10.15446/gc

Lorente, M.A. 1986. Palynology and palynofacies of the Upper Tertiary in Venezuela. Dissertationes Botanicae, 99:1-224.

Lovejoy, N.R.; Albert, J.S. \& Crampton, W.G.R. 2006. Miocene marine incursions and marine/freshwater transitions: Evidence from Neotropical fishes. Journal of South American Earth Sciences, 21:5-13. doi:10.1016/j.jsames.2005.07.009

Maia, R.G.; Godoy, H.K.; Yamaguti, H.S.; Moura, P.A.; Costa, F.S.; Holanda, M.A. \& Costa, J. 1977. Projeto de Carvão no Alto Solimões. Manaus, DNPM/CPRM, 137 p.

Muller, J.; Di Giacomo, E. \& van Erve, A. 1987. A palynologic zonation for the Cretaceous, Tertiary and Quaternary of Northern South America. Palynology, 19:7-76.

Regali, M.S.; Uesugui, N. \& Santos, A. 1974. Palinologia dos sedimentos Meso-Cenozoicos do Brasil (II). Boletim Técnico da Petrobrás, 17:263-362.

Savary, J. \& Guex, J. 1999. Discrete biochronological scales and Unitary Associations: Description of the BioGraph computer program. $1^{\mathrm{a}}$ ed. Lausanne, Mémoires de Géologie, 282 p.

Shephard, G.E.; Müller, R.D.; Liu, L. \& Gurnis, M. 2010. Miocene drainage reversal of the Amazon River driven by plate-mantle interaction. Nature Geoscience, 3:870-875. doi:10.1038/ngeo1017

Silva, S.A.F. 2004. Palinologia do Neógeno da Amazônia Sul Ocidental, Brasil. Programa de Pós-graduação em Biologia Tropical e Recursos Naturais, Instituto Nacional de Pesquisas da Amazônia, Universidade Federal do Amazonas, Dissertação de Mestrado, $106 \mathrm{p}$.

Silva-Caminha, S.A.F.; Jaramillo, C.A. \& Absy, M.L. 2010. Neogene palynology of the Solimões Basin, Brazilian Amazonia. Palaeontographica, Abt.B, 284:13-79. doi:0375-0299/10/0284-0013

Traverse, A. 1988. Paleopalynology. $1^{\text {a }}$ ed. New York, Academic Press, $813 \mathrm{p}$.

Received in May, 2016; accepted in November, 2016. 
Apêndice 1. Lista de espécies citadas neste trabalho.

Appendix 1. List of species cited on this work.

Alnipollenites verus Potonié (1931)

Bombacacidites araracuarensis Hoorn (1994B)

Bombacacidites baculatus Muller et al. (1987)

Bombacacidites fossulatus Silva-Caminha et al. (2010)

Cichoreacidites longispinosus (Lorente, 1986) Silva-caminha et al. (2010)

Corsinipollenites oculusnoctis (Thiergart, 1940) Nakoman (1965)

Crassiectoapertites columbianus (Dueñas, 1980) Lorente (1986)

Crassoretitriletes vanraadshooveni Germeraad et al. (1968)

Echinatisporis muelleri (Regali et al., 1974) Silva-Caminha et al. (2010)

Echitricolporites mcneillyi Germeraad et al. (1968)

Echitricolporites spinosus van der Hammen (1956B)

Fenestrites spinosus van der Hammen (1956B)

Grimsdalea magnaclavata Germeraad et al. (1968)

Ladakhipollenites? caribbiensis (Muller et al., 1987) Silva-Caminha et al. (2010)

Malvacipolloides sp.2 em Silva-Caminha et al. (2010)

Malvacipolloides? sp.3 em Silva-Caminha et al. (2010)

Margocolporites vanwijhei Germeraad et al. (1968)

Multimarginites vanderhammeni Germeraad et al. (1968)

Paleosantalaceaepites sp.1 em Silva-Caminha et al. (2010)

Polypodiisporites scabraproximatus Silva-Caminha et al. (2010)

Polypodiisporites? planus Silva-Caminha et al. (2010)

Pteridaceoisporis 'lobatus'Espécie informal.

Ranunculacidites operculatus (van der Hammen \& Wijmstra, 1964) Jaramillo \& Dilcher (2001)

Retitriletes altimuratus Silva-Caminha et al. (2010)

Rhoipites hispidus (van der Hammen \& Wijmstra, 1964) Jaramillo \& Dilcher (2001)

Siltaria dilcheri Silva-Caminha et al. (2010)

Tetracolporopollenites transversalis (Dueñas, 1980) Jaramillo \& Dilcher (2001) 